\section{Assessing the incidence of anti-NMDAR encephalitis}

\author{
Maxwell Greene, Eric Lancaster
}

Wright et $a l^{1}$ report the first prospective surveillance study of anti-N-methyl-Daspartate receptor (anti-NMDAR) encephalitis in children. Patients with antiNMDAR encephalitis usually have memory deficits, behavioural change and psychosis early in the disease course. ${ }^{2}$ Later they may become catatonic or comatose with central respiratory failure, bizarre writhing movements of the face or limbs, and/or profound autonomic instability. Seizures may occur at any point in the disease course. Diagnosis is confirmed by detection of antibodies to the NR1 subunit of the NMDAR. With appropriate immunotherapy many patients have a good recovery, even though this may take months. A minority of patients may relapse, but these relapses also tend to respond to treatment. Immunotherapy may include intravenous immunoglobulin, plasma exchange (PLEX), rituximab, cyclophosphamide or other treatments. In this disease, antibodies to the NMDAR receptor cause cross-linking and internalisation of the receptors and decreased NMDAR-mediated synaptic currents. Intrathecal production of antibodies may be important to pathogenesis.

Department of Neurology, The University of Pennsylvania, Philadelphia, Pennsylvania, USA

Correspondence to Dr Eric Lancaster, Department of Neurology, The University of Pennsylvania, 3400 W Spruce St., 3 W. Gates Building, Philadelphia, PA 19104, USA; eric.lancaster@uphs.upenn.edu
Anti-NMDAR encephalitis was first described in women with ovarian teratoma but men are less frequently affected. Children of either sex have increasingly been diagnosed; one large series reported that $37 \%$ of patients were under 18 years of age. ${ }^{3}$ Prior series of paediatric patients have reported more atypical presentations (speech difficulties, developmental regression, movement problems) in children compared with the typical adult presentation with psychosis and behavioural symptoms. $^{3} 4$

The incidence of anti-NMDAR encephalitis has not previously been prospectively measured, but it may be the most common cause of encephalitis in patients under 30 years of age. Wright et $a l^{1}$ have found an incidence of 0.85 per million children per year in the UK, however, the voluntary reporting system used in this study may underestimate the true incidence of the disease. Also, it is unknown how many patients may have partial manifestations of the illness that do not result in testing, and thus go undiagnosed. It is unclear if the incidence of anti-NMDAR encephalitis varies seasonally or from year to year.

In the series of Wright et al, ${ }^{1} 45 \%$ of patients were non-Caucasian. Previous studies have similarly found anti-NMDAR encephalitis among children of diverse racial and ethnic backgrounds. ${ }^{3}$ While the clinical presentations were similar across racial and ethnic groups, the frequency of teratoma is somewhat higher in Asians or African-Americans compared with Caucasians or Hispanics. ${ }^{3}$

None of the children in the study of Wright et $a l^{1}$ were reported to have tumours. About 38\% of patients with anti-NMDAR encephalitis have tumours (especially women from 12 years to 45 years of age); tumours in patients under age 12 years, or in men, are rare. ${ }^{34}$ Ovarian teratomas account for about 94\% of these tumours, although teratoma in other locations and other tumour types may rarely occur, mostly in adults. Women of Asian and African descent are somewhat more likely to have tumours than Caucasian or Hispanic women. In children with anti-NMDAR encephalitis, using tests such as MRI of the abdomen and pelvis, chest X-ray, pelvic ultrasound (in women) and (in men) testicular ultrasound is necessary to exclude tumours at the time of diagnosis. Some teratomas are too small to be identified in initial screening tests and follow-up pelvic MRI several months later may be helpful for identifying these tumours.

Anti-NMDAR encephalitis is monophasic in most patients, but may relapse. These relapses typically respond to additional immunotherapy. The risk of relapse may be lower in patients treated with immunotherapies, especially the second-line therapies (cyclophosphamide, rituximab). ${ }^{3}$ The $23 \%$ relapse rate in paediatric patients for the study of Wright et al is consistent with the numbers from prior studies. ${ }^{4}$ These numbers highlight the importance of ongoing care for these patients and prompt evaluation of any new or worsening symptoms. It is an open question whether patients who respond well to first-line immunotherapy should be treated with a second agent, such as rituximab, to prevent relapse. 
First-line therapy for anti-NMDAR encephalitis usually involves steroids, intravenous immunoglobulin, and/or PLEX. Symptoms generally improve with treatment, but it is unknown which immunotherapies are most effective. Titulaer et $a l^{3}$ in their large retrospective study of anti-NMDAR encephalitis, have noted that the severity of illness influences physician treatment decisions and confounds determination of the degree of efficacy for individual treatments. While PLEX effectively decreases serum antibodies, it is unclear how effectively PLEX decreases antibody levels in the central nervous system (CNS), which may be more important for disease pathogenesis. PLEX can also remove monoclonal antibody therapies such as rituximab. For these reasons some practitioners have favoured intravenous immunoglobulin. However, Wright et $a l^{1}$ showed an interesting correlation in their patients between PLEX treatment and full recovery at 12 months (total $n=9$ ), suggesting that decreasing peripheral NMDAR antibodies may be helpful. This finding must be interpreted cautiously as it may be confounded by other first-line treatments used concurrently $(n=2)$, or the higher number of patients who also received second-line therapies in addition to PLEX $(n=7)$. Also, Titulaer et al's ${ }^{3}$ statistical analysis of over 500 patients with antiNMDAR encephalitis suggests that patients who receive PLEX are treated earlier after symptom onset, which is an independent predictor of a favourable outcome. However, if this result is confirmed, ideally with a prospective randomised treatment trial, PLEX should be more consistently used as a first-line therapy.

In addition to NMDAR, at least 10 other CNS synaptic proteins are targets of distinct autoantibody syndromes. These targets include ionotropic receptors (AMPA receptor, GABA-A receptor, Glycine receptor), metabotropic receptors (GABA-B receptor, mGluR1, mGluR5), voltage-gated ion channels (dipeptidyl-peptidase-like protein-6, voltage-gated calcium channel) and ionchannel-associated proteins (LGI1, Caspr2). ${ }^{5}$ Some of these autoantibody disorders have characteristic clinical syndromes and/or cancer associations, but most could present with encephalitis and behavioural change. While anti-NMDAR encephalitis may be the most common of these disorders, particularly in children, testing for these different aetiologies of autoimmune encephalitis should not be overlooked in the differential, as the prognosis and treatment may differ significantly. The true incidence of these diseases in different populations has not been prospectively studied. In the future, reliable testing for panels of autoantibodies, perhaps informed by specific clinical phenotypes, may be more fully implemented in clinical practice.

Since the discovery of anti-NMDAR encephalitis in 2007 the ability of physicians to recognise and diagnose this disorder has increased tremendously. Additional work will be required to fully define the scope of the disease worldwide, the yield of testing in various clinical scenarios, the best methods for detecting tumours and the optimal treatments. The study by Wright et al has added to this body of knowledge by being the first to prospectively examine the incidence of this disease.
Funding Dana Foundation (Neuromimmunology Award), National Institute of Neurological Disorders and Stroke (K08-NS-075142-01A1).

Competing interests $\mathrm{EL}$ received payment from Grifols for teaching a course and provided paid expert opinions for the US Division of Vaccine Injury Compensation.

Provenance and peer review Commissioned; internally peer reviewed.

To cite Greene M, Lancaster E. Arch Dis Child 2015;100:512-513.

Received 9 February 2015

Accepted 5 March 2015

Published Online First 25 March 2015

\section{SLinked}

http://dx.doi.org/10.1136/archdischild-2014-306795

Arch Dis Child 2015;100:512-513.

doi:10.1136/archdischild-2014-307978

\section{REFERENCES}

1 Wright S, Hacohen $\mathrm{Y}$, Jacobson L, et al. N-methyl-Daspartate receptor antibody-mediated neurological disease: results of a UK-based surveillance study in children. Arch Dis Child 2015;100:521-6.

2 Dalmau J, Lancaster E, Martinez-Hernandez E, et al. Clinical experience and laboratory investigations in patients with anti-NMDAR encephalitis. Lancet Neurol 2011:10:63-74.

3 Titulaer MJ, McCracken L, Gabilondo I, et al. Treatment and prognostic factors for long-term outcome in patients with anti-NMDA receptor encephalitis: an observational cohort study. Lancet Neurol 2013;12:157-65.

4 Florance NR, Davis RL, Lam C, et al. Anti-N-methyl-Daspartate receptor (NMDAR) encephalitis in children and adolescents. Ann Neurol 2009;66:11-18.

5 Lancaster E, Dalmau J. Neuronal autoantigenspathogenesis, associated disorders and antibody testing. Nat Rev Neurol 2012:8:380-90. 